\title{
Reconstruction and Analysis Software Environment of LHCb
}

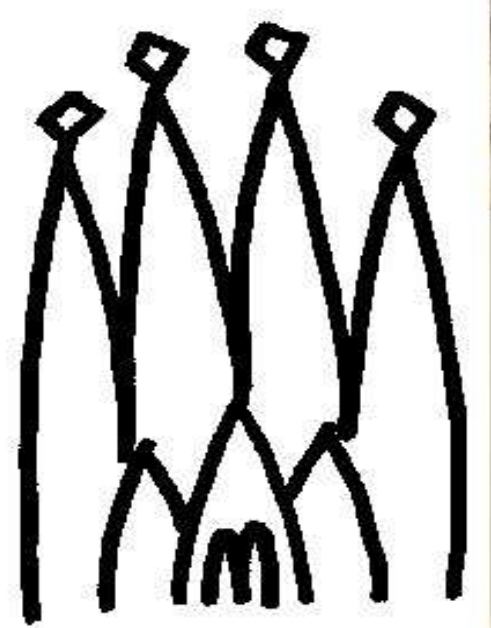

- LHCb reconstruction and analysis

- Software Architecture "Gaudi"

- Some Examples:

- High-Level Trigger

- Event Display

- Interactive Analysis
Patrick Koppenburg

CERN / PH

On behalf of the LHCb collaboration 


\title{
Software Environment of LHCb
}

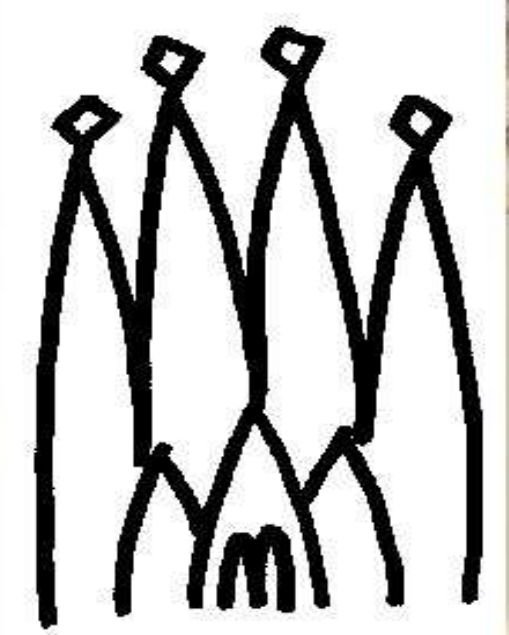

- LHCb reconstruction and analysis

- Software Architecture "Gaudi"

- Some Examples:

- High-Level Trigger

- Event Display

- Interactive Analysis

\author{
Patrick Koppenburg \\ CERN / PH \\ On behalf of the LHCb collaboration
}




\section{LHCb Software strategy}

Structure: Develop an Architecture ("blueprint") and a Framework

(real code) to be used at all stages of LHCb data processing

- Software triggers, simulation, reconstruction, analysis, visualization...

- One single framework used by all members of the collaboration for all code

$\rightarrow$ Gaudi

Development: Avoid duplication of computing effort

- Develop simple components that can be used in any environment

- Use common interfaces to allow easy "plug-and-play" switching of tools. Ex: Vertex Fitters, Cuts...

Applications are developed by customizing the framework. 


\section{Gaudi Architecture}

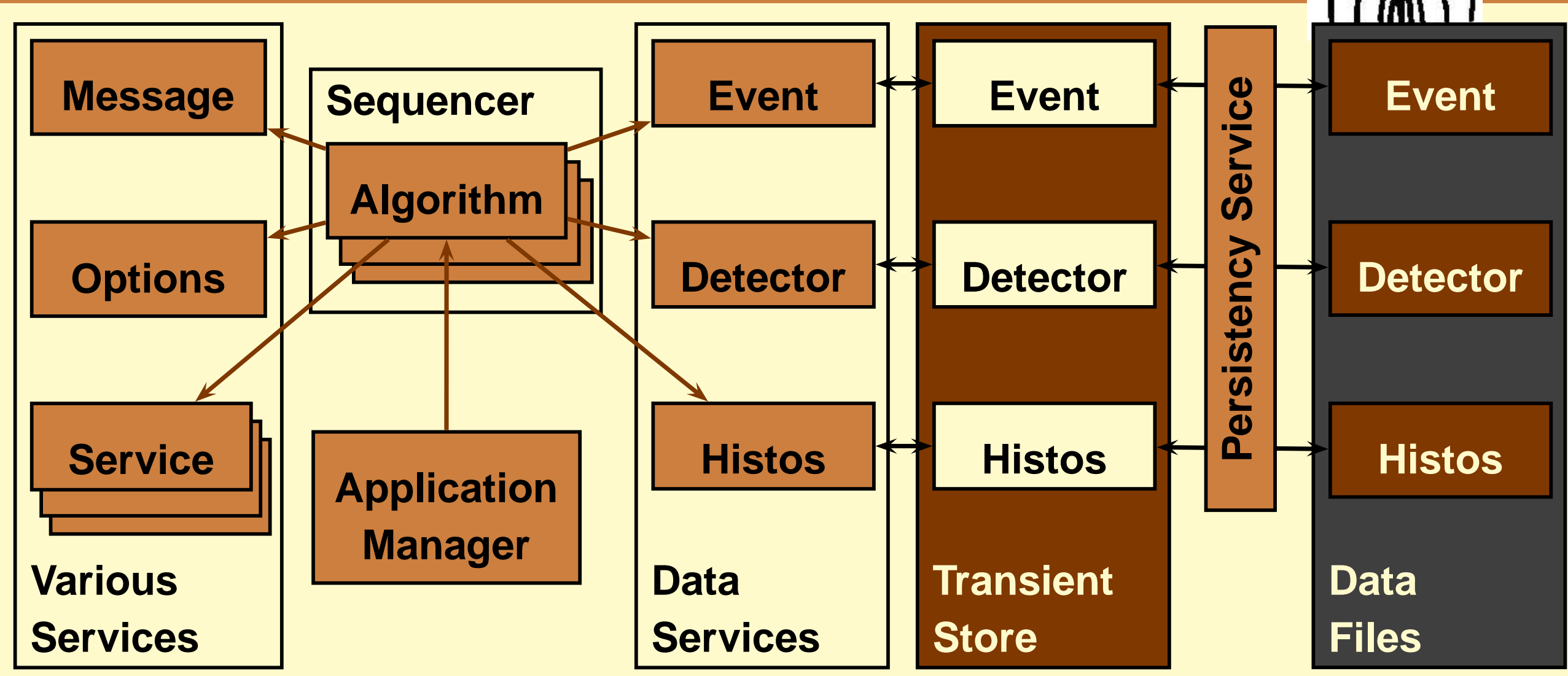

- Separation of "data" and "algorithms": Write 00 code, but profit from decades of HEP experience

- Separation of "transient" and "persistent" data

- Physicist code encapsulated in specific places (Algorithms) 


\section{Gaudi Architecture}

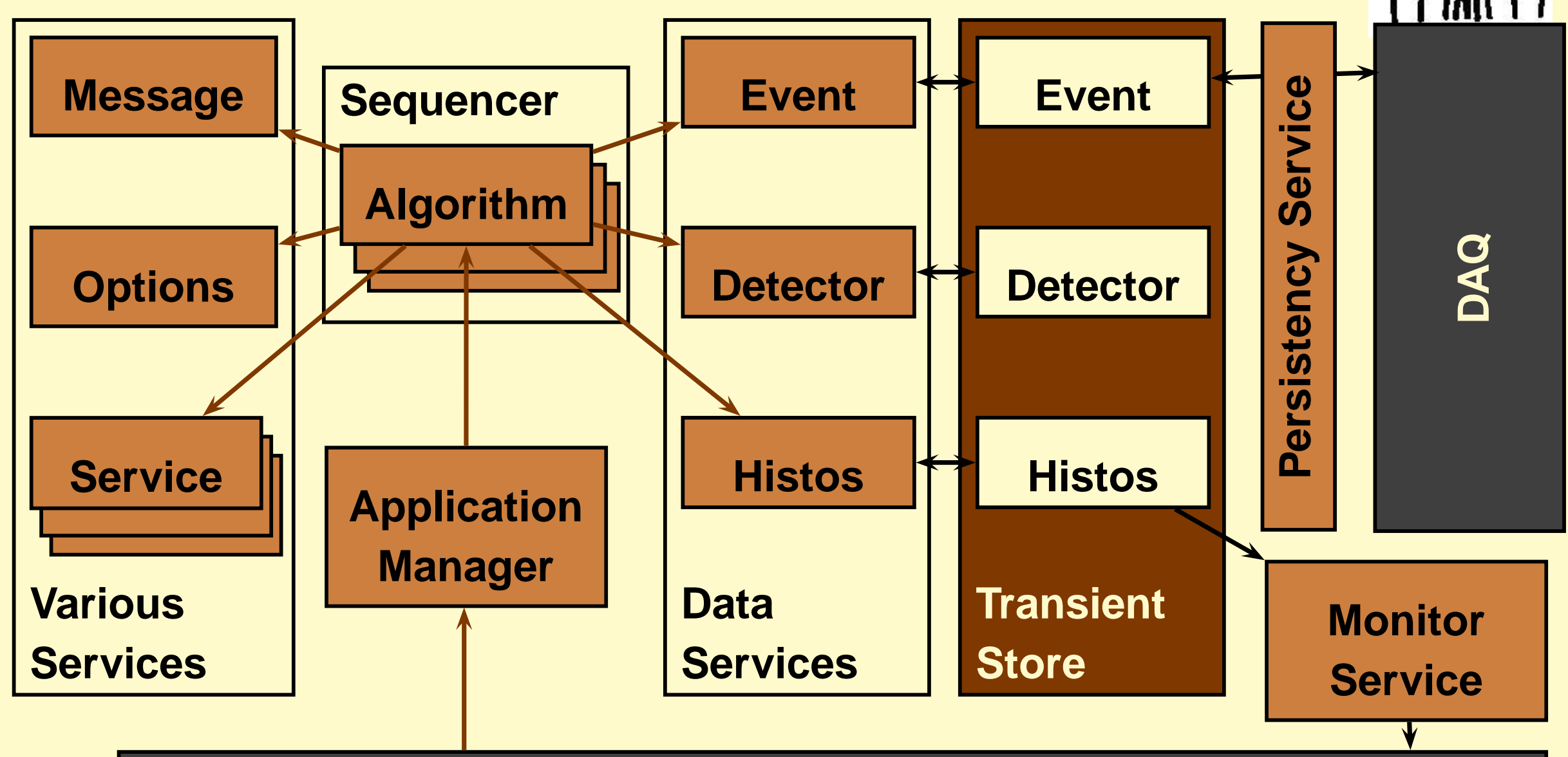

\section{Experiment Control System}

The same architecture is used online in the software triggers $\rightarrow$ The same algorithms are used 


\section{Example: High-Level Trigger}

Mins

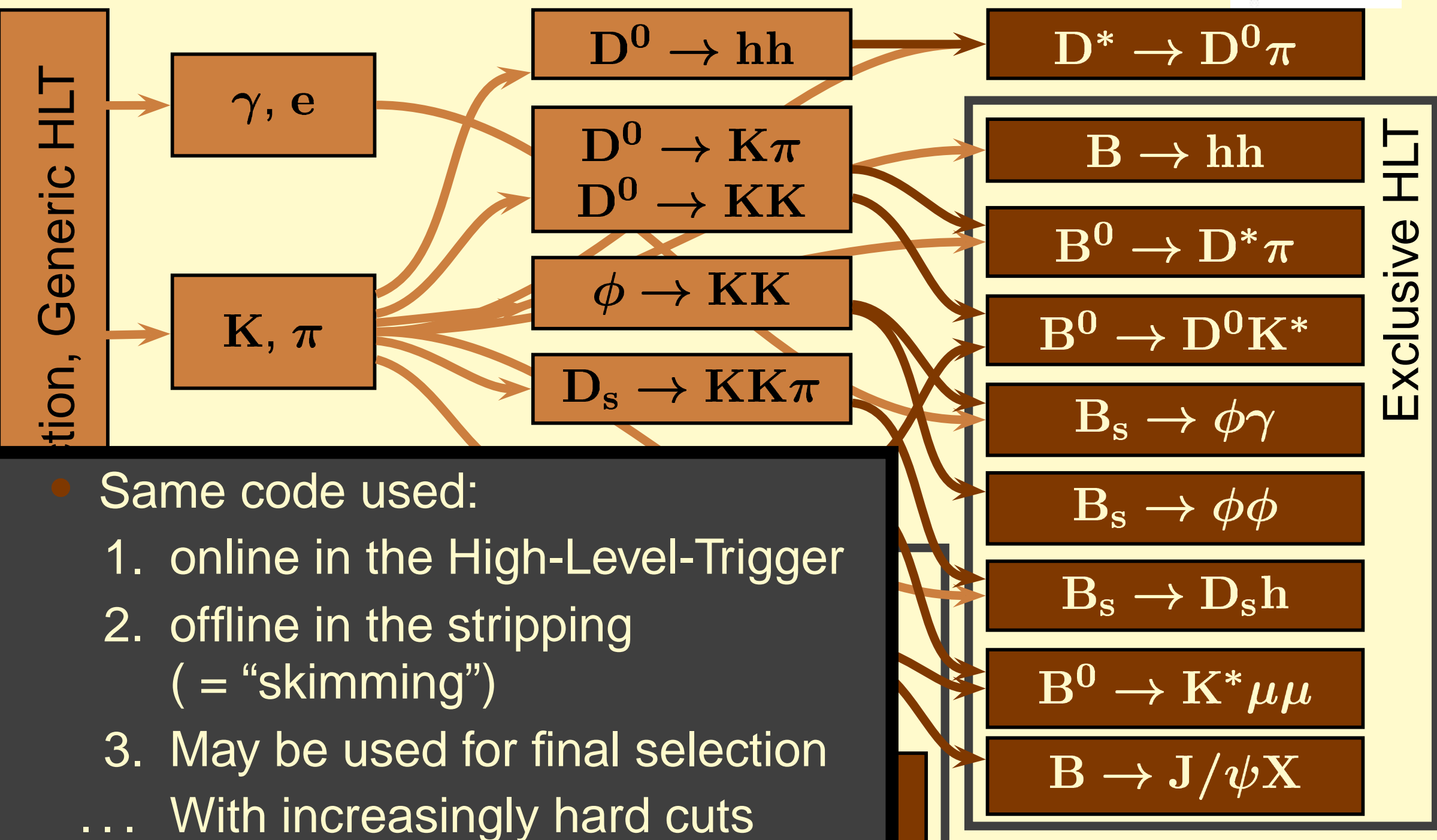

$\rightarrow$ Maximal correlations of selections 


\section{Applications}

Gauss: Simulation. Uses generators and Geant 4.

011010011101

10101000101

$01010_{110100}$ Boole

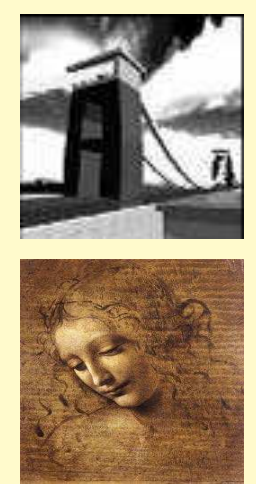

.
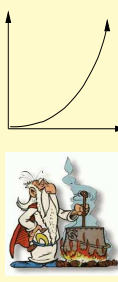

Boole: Digitization. Simulates detector response and transforms to "raw" data format.

Brunel: Reconstruction.

Full pattern recognition and PID.

DaVinci: Analysis. Deals with "Particles" and "Vertices". Final event selection.

Euler: L1 Trigger. For on- and offline use.

Moore: High-Level Trigger. For on- and offline use.

Panoramix: Visualization.

Bender: Interactive Analysis in python. 


\section{Data Production Flow}
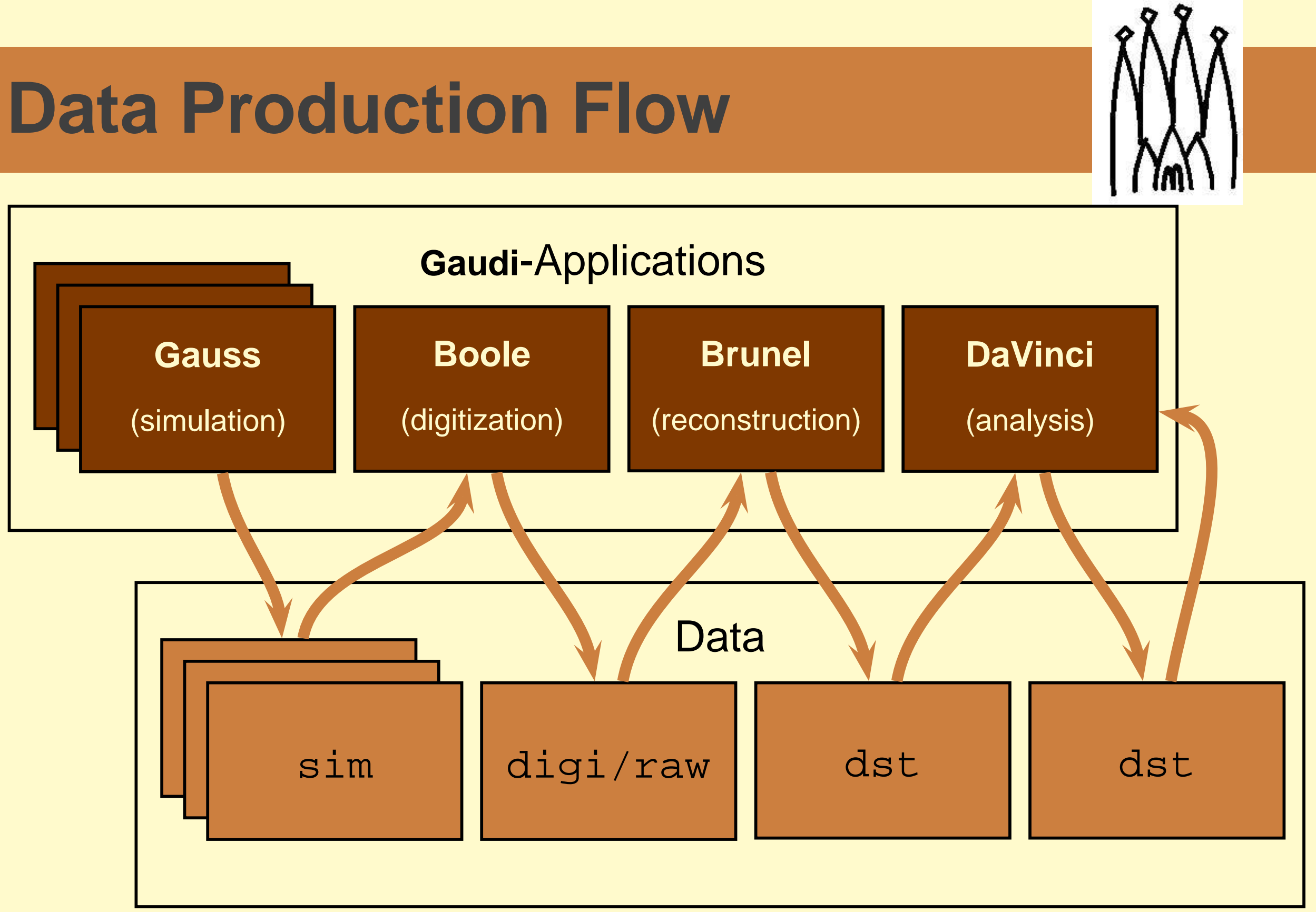

$\sim 400$ million events produced during 2004 data challenge 


\section{Example: Panoramix Display}
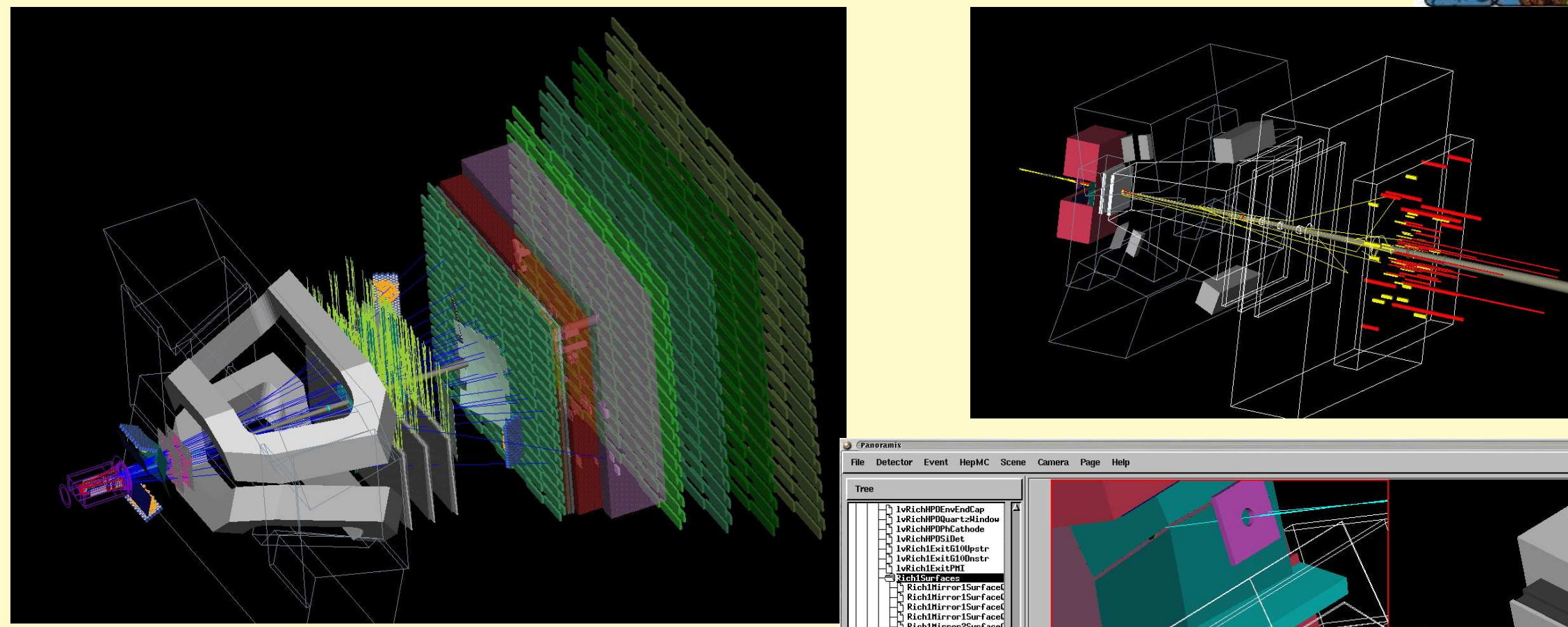

- Visualization application based on Gaudi, OnX and OpenInventor

- Scripting based on python

- Allows to (re-)process ev-
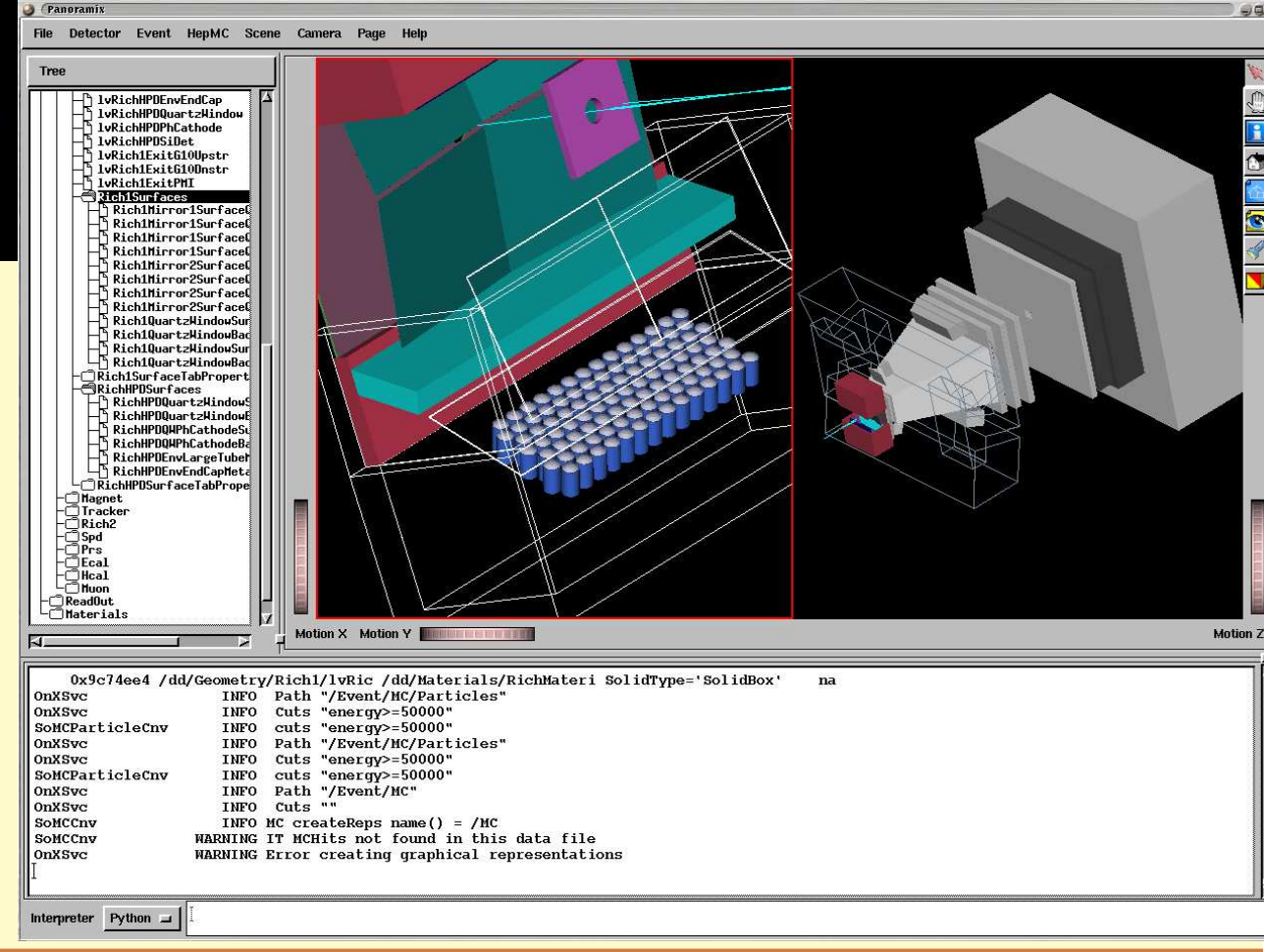


\section{Example: Interactive analysis}

\section{Min}

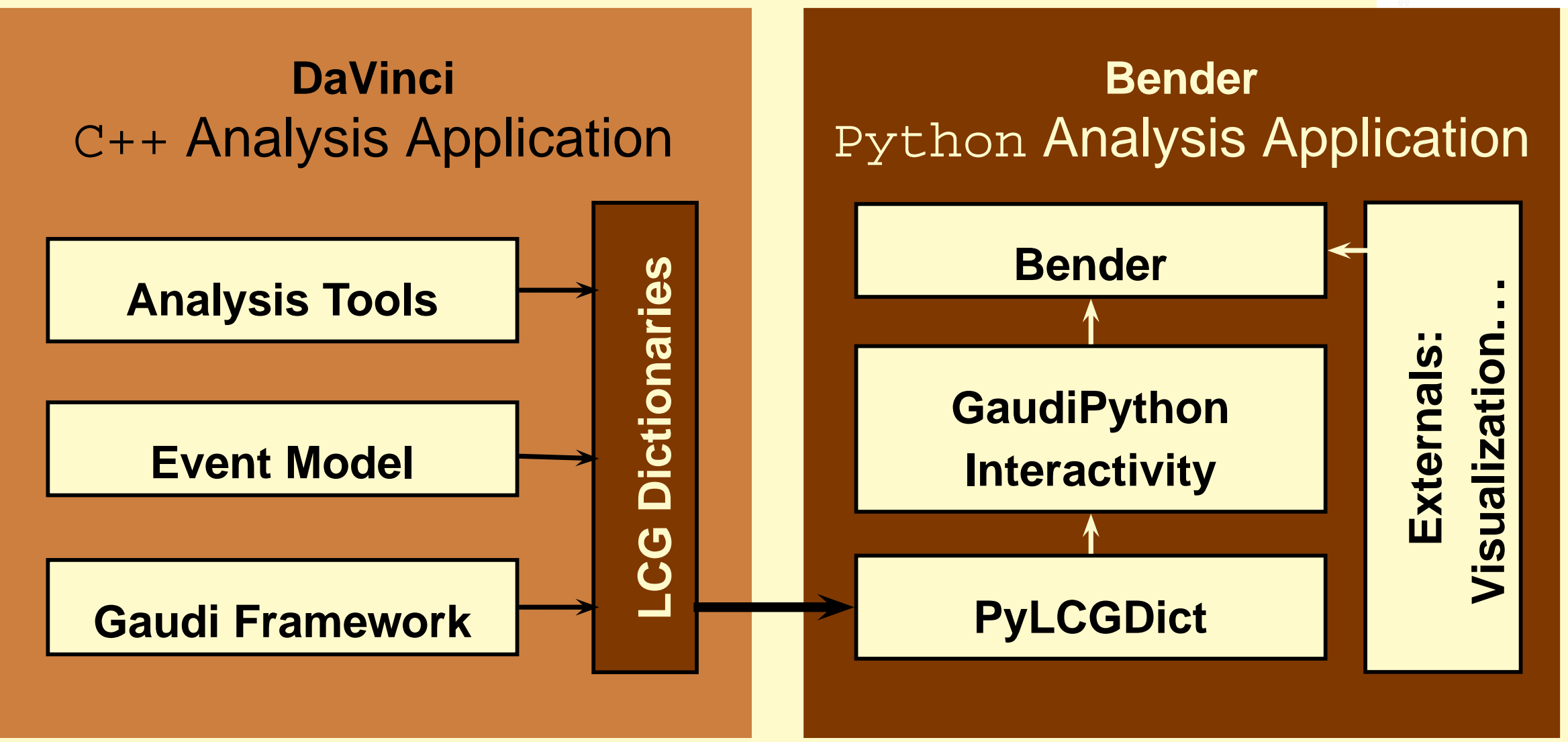

- Bender combines a python-wrapping of the DaVinci tools, the LHCb Event Model and the Gaudi framework

- It allows to perform interactive physics analysis...

- ... and access to many external tools. 


\section{Example: Interactive analysis}
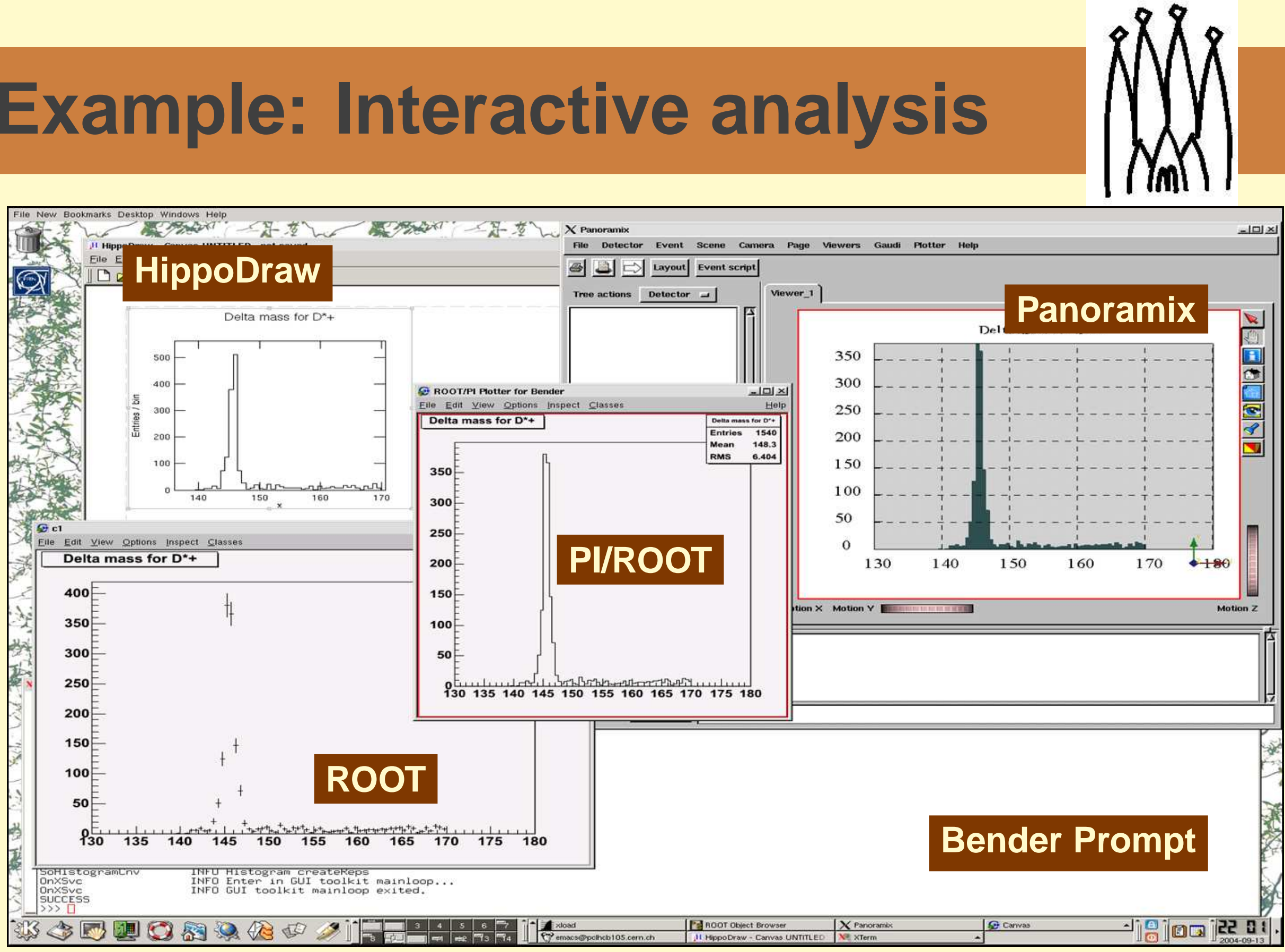


\section{Conclusion}

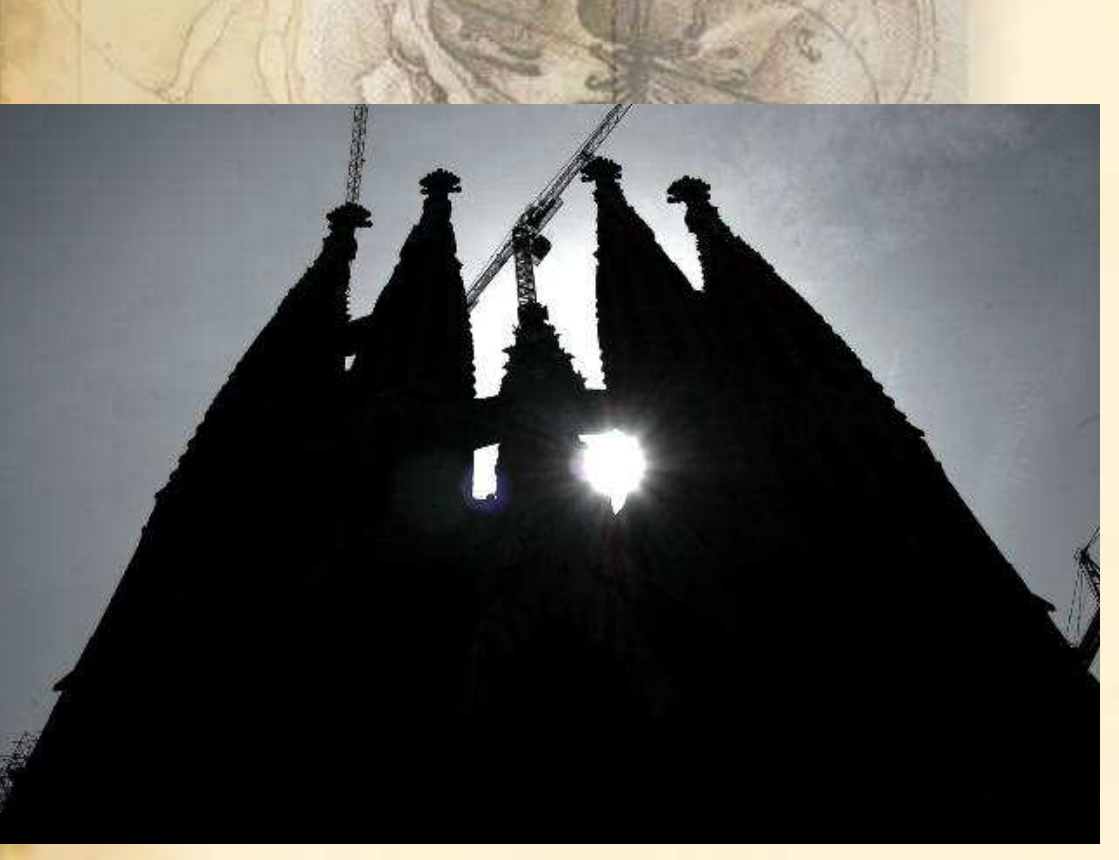

- A well defined structure for 500 users

- One framework for 8 applications:

- Data production

- Analysis

- Trigger

- Interactivity

- Facilitates migration of algorithms between applications

- Encourages optimal usage of the code

- Helps to minimize inefficiencies

- Allows analysis in $\mathrm{C}++$ or python 